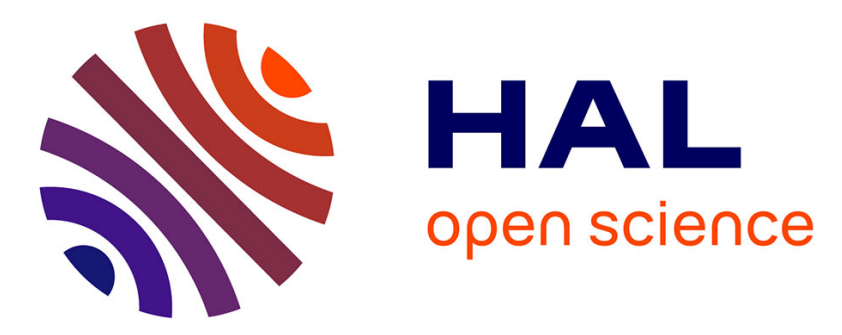

\title{
Direct and correlated responses to selection in a host-parasite system: testing for the emergence of genotype specificity \\ Thibault Nidelet, Oliver Kaltz
}

\section{- To cite this version:}

Thibault Nidelet, Oliver Kaltz. Direct and correlated responses to selection in a host-parasite system: testing for the emergence of genotype specificity. Evolution - International Journal of Organic Evolution, 2007, 61 (8), pp.1803-1811. 10.1111/j.1558-5646.2007.00162.x . hal-02901373

\section{HAL Id: hal-02901373 \\ https://hal.inrae.fr/hal-02901373}

Submitted on 17 Jul 2020

HAL is a multi-disciplinary open access archive for the deposit and dissemination of scientific research documents, whether they are published or not. The documents may come from teaching and research institutions in France or abroad, or from public or private research centers.
L'archive ouverte pluridisciplinaire $\mathbf{H A L}$, est destinée au dépôt et à la diffusion de documents scientifiques de niveau recherche, publiés ou non, émanant des établissements d'enseignement et de recherche français ou étrangers, des laboratoires publics ou privés. 


\title{
DIRECT AND CORRELATED RESPONSES TO SELECTION IN A HOST-PARASITE SYSTEM: TESTING FOR THE EMERGENCE OF GENOTYPE SPECIFICITY
}

\author{
Thibault Nidelet ${ }^{1,2}$ and Oliver Kaltz ${ }^{1,3}$ \\ ${ }^{1}$ Laboratoire de Parasitologie Evolutive (CC 237), CNRS-UMR 7103, Université Pierre et Marie Curie, 7 quai St. Bernard, \\ 75252 Paris Cedex 05, France \\ ${ }^{2}$ E-mail: Thibault.nidelet@snv.jussieu.fr \\ ${ }^{3} E$-mail: okaltz@snv.jussieu.fr
}

Received December 13, 2006

Accepted March 16, 2007

\begin{abstract}
Genotype $\times$ environment interactions can facilitate coexistence of locally adapted specialists. Interactions evolve if adaptation to one environment trades off with performance in others. We investigated whether evolution on one host genotype traded off with performance on others in long-term experimental populations of different genotypes of the protozoan Paramecium caudatum, infected with the bacterial parasite Holospora undulata. A total of nine parasite selection lines evolving on three host genotypes and the ancestral parasite were tested in a cross-infection experiment. We found that evolved parasites produced more infections than did the ancestral parasites, both on host genotypes they had evolved on (positive direct response to selection) and on genotypes they had not evolved on (positive correlated response to selection). On two host genotypes, a negative relationship between direct and correlated responses indicated pleiotropic costs of adaptation. On the third, a positive relationship suggested cost-free adaptation. Nonetheless, on all three hosts, resident parasites tended to be superior to the average nonresident parasite. Thus genotype specificity (i.e., patterns of local adaptation) may evolve without costs of adaptation, as long as direct responses to selection exceed correlated responses.
\end{abstract}

KeY WORDS: Cost of adaptation, experimental evolution, generalists, Holospora undulata, local adaptation, Paramecium caudatum, specialists.

Diversifying selection in heterogeneous environments can produce and maintain genetic diversity (Levins 1968). Genotype $\times$ environment interactions are a signature of this process, and ideally, they allow the coexistence of locally adapted specialists (genotypes or species) (Futuyma and Moreno 1988; Kassen 2002; Kawecki and Ebert 2004). It is commonly assumed that these interactions evolve if adaptation to one environment trades off with performance in other environments (Kawecki and Ebert 2004).

The precise shape of genotype $\times$ environment interactions depends on the relative magnitude of the direct and correlated re- sponses to selection (Fig. 1). From the above common view, we predict a positive direct response in the selection environment and a negative correlated response in environments in which a genotype has not been selected. This results in crossing reaction norms (= $\mathrm{G} \times \mathrm{E}$ interactions) and local adaptation (Fig. 1A, but see also Fig. 1B). Furthermore, a negative association between direct and correlated responses to selection is expected if costs of adaptation are caused by antagonistic pleiotropy, that is, if genes improving fitness in the selection environment produce a proportional decrease in other environments (Levins 1968; MacLean and Bell 2002). 

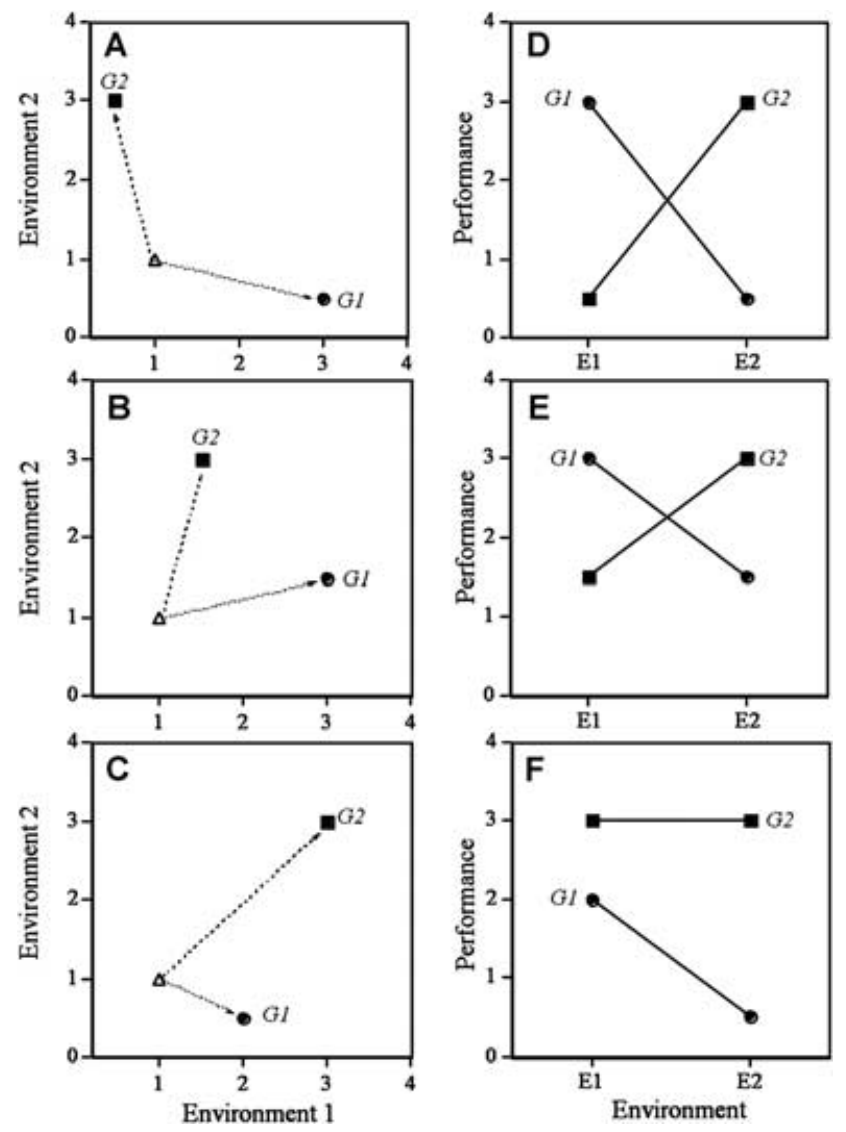

Figure 1. Potential relationships between the response to selection and the evolution of genotype $\times$ environment interactions and specialization to two hypothetical environments (adapted from [Bell 1997, p. 352]). (A-C) Performance of genotypes after selection in either environment 1 or environment 2 (closed symbols). Arrows describe the advance or regress relative to the ancestral genotype (triangle). (D-F) Reaction norms of the evolved genotypes in the two environments. (A, D) Standard cost-of-adaptation scenario: (A) Advance in the selection environment relative to the ancestor (positive direct response to selection, DR), regress in the other environment (negative correlated response to selection, CR), resulting in crossing reaction norms and local adaptation (D). Note that the negative CR can be due to antagonistic pleiotropy and/or accumulation of conditionally deleterious mutations (Kassen 2002). (B, E) Advance in both environments (positive DR and CR). The DR exceeds the CR in both environments (B), resulting in crossing reaction norms and local adaptation (E), despite the absence of costs of adaptation. For theoretical formalizations of this scenario, see Whitlock 1996; Kawecki 1998. (C, F) Positive DR in both environments, but strong positive $C R$ in environment 1 (C). Absence of costs of adaptation for genotypes evolving in environment 2 results in universally superior generalist (F).

However, experimental evolution studies suggest that the evolution of trade-offs is by no means the inevitable outcome of selection (reviewed in Kassen 2002). In fact, whether costs of adaptation arise often depends on both the selection environment and the environment in which the correlated response is measured (Kassen 2002; MacLean and Bell 2002). Clearly, if the expression of costs is variable, the ideal picture of perfectly symmetric $\mathrm{G} \times$ $E$ interactions, with each environment occupied by its respective local specialist, is no longer valid. Instead, populations may harbor only a few dominant genotypes, or, at the extreme, a single, universally superior generalist (Fig. 1C).

We investigated the costs of adaptation and evolution of genotype $\times$ environment interactions in a host-parasite system. Here, the environment of the parasite is the host, and vice versa. In many systems, infection success is determined by lock-and-key recognition mechanisms (Thompson and Burdon 1992; Frank 1994; De Wit 1997; Schmid-Hempel and Ebert 2003). It is conceivable that evolving the right key (e.g., the presence or absence of an elicitor molecule) to infect a particular host genotype represents the wrong key on other hosts with different locks (e.g., receptor profiles). Thus, as for abiotic environments, costs of adaptation may arise and lead to host genotype $\times$ parasite genotype interactions characterized by gene-for-gene or matching-allele systems (Frank 1994; Agrawal and Lively 2002; Dybdahl and Storfer 2003). These interactions are considered as a basic ingredient of the coevolutionary process as they can generate recurrent cycles of frequency-dependent selection (Agrawal and Lively 2002, and references therein) and thereby maintain genetic diversity in parasite and host. In subdivided populations, costs of adaptation may translate into patterns of local adaptation of parasites to their hosts, or vice versa (Morand et al. 1996; Kaltz and Shykoff 1998).

Genotype $\times$ genotype interactions (Lambrechts et al. 2006) and local adaptation (Kaltz and Shykoff 1998; Bergelson et al. 2001; Lajeunesse and Forbes 2002) have been demonstrated for naturally occurring genotypes in various systems, but only a few studies have investigated how these patterns evolve (e.g., Gould 1979; Karban 1989; Fry 1990; Ebert 1998; Agrawal 2000; Turner and Elena 2000; Buckling and Rainey 2002). Costs of adaptation seem to shape the coevolutionary process at least to some extent. Serial passage of parasites on novel host species sometimes leads to reduced performance on the original host (Ebert 1998), although not necessarily on other novel host species (Gould 1979; Agrawal 2000). At the within-species level, the evolution of trade-offs and concomitant genotype-specific adaptation has been demonstrated for eukaryotic (Karban 1989) as well as microbial (Turner and Elena 2000; Buckling and Rainey 2002) systems.

We investigated the direct and correlated responses to selection of the bacterial parasite Holospora undulata to its protozoan host, Paramecium caudatum. This parasite infects the micronucleus of its host and can be transmitted both vertically and horizontally (Kaltz and Koella 2003; Fokin 2004; Restif and Kaltz 2006). We maintained infected and uninfected replicate populations of three host clones for 12 months. Then a cross-infection experiment was carried out to measure infection success of evolved 
and ancestral parasites on each host clone. We used hosts from the uninfected control populations and therefore did not consider adaptive changes in the host. The following questions were addressed: (1) Does the parasite adapt to the novel host genotypes (positive direct response to selection)? (2) Are there costs of adaptation (negative correlated response to selection)? (3) What is the relationship between direct and correlated responses to selection, and does it vary among parasites evolving on different host clones? (4) Are there host genotype $\times$ parasite genotype interactions and do parasites specialize on the host genotypes with which they coevolve?

\section{Materials and Methods STUDY ORGANISMS}

The freshwater ciliate $P$. caudatum feeds on bacteria and detritus ingested from the water column. Reproduction is predominantly asexual by mitotic division; the sexual cycle (conjugation of cells of opposite mating type) is induced by environmental stress. Paramecia have a polyploid macronucleus, where genes are expressed during the asexual cycle, and a diploid micronucleus mostly active during the sexual cycle (Wichtermann 1986; Görtz 1988).

The gram-negative bacterium $H$. undulata ( $\alpha$-group of the Protobacteria [Amann et al. 1991]) is a natural parasite of P. caudatum. Infection starts with the ingestion of infectious forms (15$20 \mu \mathrm{m}$ ) during food uptake. The parasite then escapes from the digestive vacuole and is transferred to the micronucleus. Infectious forms differentiate into reproductive forms $(5 \mu \mathrm{m})$, which multiply and begin to fill up the nucleus. Vertical transmission occurs when bacteria segregate into the daughter nuclei of the mitotically dividing host (Görtz and Dieckmann 1980). Accumulation of reproductive stages induces their differentiation into infectious forms after 7-10 days (Fels and Kaltz 2006). Subsequently, up to several hundred infectious forms can be observed in the heavily swollen micronucleus. At this stage, infection reduces host division and survival (Restif and Kaltz 2006). Infectious forms are released into the environment upon host death, but to some degree also during host division (Wiemann and Görtz 1989).

\section{LONG-TERM EXPERIMENT}

In October 2002, we established separate mass cultures of nine host clones from different geographic origins (Restif and Kaltz 2006). Each clonal mass culture was derived from a single individual, then split and one half inoculated with parasites from an infected base culture (prevalence: $90-100 \%$ ). Both host and parasite from this base culture were originally collected in Germany and provided by H.-D. Görtz (University of Stuttgart, Germany). Infected and uninfected cultures were then further split into three to six replicate lines, each consisting of $50 \mathrm{~mL}$ (2000-5000 parame- cia), kept in $50 \mathrm{~mL}$ Falcon tubes. The culture medium was made of Protozoan Pellets (Carolina Biological Supply Company, Burlington, $\mathrm{NC}$ ), suspended at a concentration of $0.7 \mathrm{~g} / \mathrm{L}$ in Volvic mineral water, autoclaved and then inoculated with the bacterium Serratia marcenscens (strain A173; Institut Pasteur, Paris, France) as food resource for the paramecia. The lines were kept at $23^{\circ} \mathrm{C}$. Once a week, $10 \mathrm{~mL}$ of the population (20\%) were removed and replaced with fresh medium inoculated with food bacteria. To preserve the parasite in its ancestral state, samples of the infected base culture were kept at $5^{\circ} \mathrm{C}$, which almost completely stops development of both host and parasite (Fokin 2004).

\section{ADAPTATION ASSAY}

In December 2003, we carried out an adaptation assay, using parasites from three (randomly chosen) host clones (K3, K4, and K8) from the long-term experiment. These host clones originate from independent crosses between two Japanese clones (KNZ 5, mating type $\mathrm{O} 3$ and KNZ 2, mating type E3, provided by T. Watanabe, Tohoku University, Japan). Assuming 10 bacterial generations (i.e., divisions of reproductive forms inside the micronucleus) per infection cycle, and 10 days to complete the cycle, this amounts to a maximum of 400 parasite generations, or 40 infection cycles, per year.

The (potentially) evolved parasites from nine replicate lines (three from each host clone), and the ancestral parasites were tested. To obtain a sufficiently large inoculum, $25 \mathrm{~mL}$ from each replicate line were regrown to a volume of $75-100 \mathrm{~mL}$. At the same time, samples of the infected founder population (still at prevalences of over $90 \%$ ) were transferred to $23^{\circ} \mathrm{C}$ and also regrown. Inocula were obtained by crushing infected cultures with a tissue homogenizer to release the infectious forms of the parasite (for details see Millot and Kaltz 2006; Restif and Kaltz 2006). The inocula were diluted to a density of 125 infectious forms per microliter.

Parasites were assayed on hosts from uninfected control replicate lines. Three weeks before the assay, we isolated one paramecium from each of three replicate lines from the clones $\mathrm{K} 3, \mathrm{~K} 4$, and K8. The nine paramecia were grown up as clonal mass cultures (referred to as "subclones," hereafter). Each evolved parasite line was tested against each host subclone $(9 \times 9=81$ replicates $)$. For the founder parasite, we established two replicates per host subclone $(2 \times 9=18$ replicates $)$. A replicate consisted of a $50 \mathrm{~mL}$ Falcon tube with 400 paramecia in a volume of $5 \mathrm{~mL}$ of diluted (20\%) growth medium to which we added $5 \times 10^{4}$ infectious forms.

Infection success was measured at three time points. A first sample of 50-60 individuals was taken 48-h post inoculation (PI) and the proportion of infected individuals determined at $1000 \times$ magnification after fixation with lacto-aceto-orcein (Görtz and Dieckmann 1980). This assay measured the parasite's capacity of 
horizontal transmission (Fels and Kaltz 2006; Lohse et al. 2006). To measure parasite fitness under conditions similar to those in the long-term selection lines, we proceeded as follows. One week PI, 200 individuals from each assay tube were transferred to a new tube with fresh medium added in two- to three-day intervals until a volume of $50 \mathrm{~mL}$ was reached around day $17 \mathrm{PI}$. This generates a logistic growth curve (Kaltz and Koella 2003) with moderate growth during the last 7-10 days, until populations reached carrying capacities comparable to those in the long-term cultures (T. Nidelet, unpubl. data). On day 25 PI, a second measurement of prevalence was taken, as described above. Medium was then renewed weekly, until a third sample was fixed on day 62 PI. Thus, assays 2 and 3 provided an integrated estimate of parasite fitness, determined by rates of horizontal and vertical transmission and by virulence (reduction in host division and/or survival), just like in the selection lines.

\section{DATA ANALYSIS}

For each combination of parasite replicate line, host subclone and assay, the response to selection was calculated as (prevalence [evolved parasite] / mean prevalence [ancestral parasite] -1 ). Thus a positive response means that evolved parasites produced more infections than did the ancestral parasites. The direct response to selection is calculated for the host clone on which the parasite evolved, the correlated response for host clones on which the parasite did not evolve. Assays were analyzed separately. First, we calculated the mean direct and correlated responses to selection for each parasite line $(\mathrm{N}=2 \times 9=18)$ to test for differences between parasite identity (K3, K4, and $\mathrm{K} 8$ ) and type of response (direct vs. correlated) in an analysis of variance (ANOVA). We also performed $t$-tests to establish whether responses to selection were significantly different from zero. Second, if adaptation to a host genotype bears pleiotropic costs, we expect a negative relationship between the direct and correlated response to selection. To test for this, we used the above dataset and carried out an analysis of covariance (ANCOVA) with the correlated response as dependent, and parasite identity and direct response as independent variables. Third, analogous to the two-environment scenarios in Figure 1, we tested for parasite identity $\times$ host clone interactions in each of the three pairwise combinations of host and parasite identities (K3-K4; K8-K4; K3-K8) by means of ANODEV (factorial models; factors: parasite identity, replicate line [identity], host clone, subclone [host clone]). Fourth, we calculated the combined prevalences of resident and nonresident parasites on each host subclone $(\mathrm{N}=18)$. We then tested for host genotype-specific adaptation (resident vs. nonresident parasite) and host clone effects in an analysis of deviance (ANODEV), based on a logistic regression with presence/absence of infection as response variable. We used the JMP (SAS 2003) and SAS (SAS 1996) statistical packages for the analysis.

\section{Results \\ OVERVIEW}

All replicate tubes were found to be infected in assay 1 (48-h $\mathrm{PI}$ ), with prevalences ranging from 0.07 to 0.82 (mean: $0.362 \pm$ $0.017 \mathrm{SE})$. One tube lost infection thereafter, and mean prevalences reached $0.43 \pm 0.02$ in assay 2 (day $25 \mathrm{PI}$ ), and $0.40 \pm$ 0.02 in assay 3 (day $62 \mathrm{PI}$ ). Consistent with previous results, the three host clones differed in average resistance to the three different parasites (Table 3; prevalences K4: $0.25 \pm 0.02$; K3: $0.38 \pm$ 0.03; K8: $0.55 \pm 0.06$, averaged over the three assay means). Mean host population size was $4457 \pm 362$ in assay 2, but only $1886 \pm$ 135 in assay 3 (four tubes died out). Prevalence in assay 2 was positively correlated with reduction in host density between assay 2 and assay 3 ( $r=0.44, \mathrm{~N}=98, P<0.0001)$, indicating that this reduction at least partly reflects parasite virulence.

Assay 2 revealed a significant positive overall direct (DR; $t_{8}=$ $3.13, P=0.0141)$ and marginally significant positive correlated (CR) response to selection (DR; CR; $t_{17}=2.14, P=0.0473$; tests based on the means per combination of parasite line and host clone). In assay 1 , both types of responses were small and not significantly different from 0 (DR: $t_{8}=-0.10$, ns; CR: $t_{17}=$ $0.53, P=\mathrm{ns}$ ), and even tended to become negative in assay 3 (DR: $t_{8}=-0.94$, ns.; CR: $t_{17}=-1.68, P=0.1113$; Fig. 2). Estimates of the response to selection were not significantly correlated between assay dates (not shown), although the ranking of the means of parasite identities was the same in assay 2 and $3(\mathrm{~K} 8>\mathrm{K} 3>$ K4). Overall, assay 2 provided the best statistical support for the variation in the response to selection (Tables 1 and 2) and for genotype-specific adaptation (Table 3). Below we will describe these patterns in more detail.

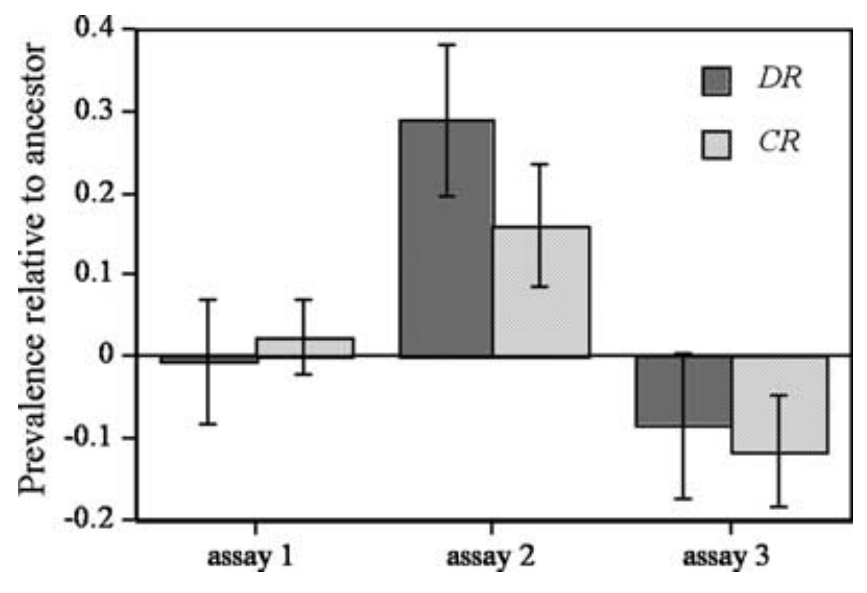

Figure 2. Mean ( $\pm 1 \mathrm{SE}$ ) direct (DR) and correlated (CR) responses to selection in assays 1-3. Positive values indicate a higher infection success of evolved parasites relative to the ancestral parasites. Averages taken over the means per parasite replicate line and host clone (DR: $N=9$; CR: $N=18$ ). 
Table 1. ANOVA of the response to selection as a function of type of response (direct or correlated, "DR/CR"), parasite identity, and parasite replicate line in the three assays. Identity was tested against replicate line, DR/CR against the DR/CR $\times$ identity interaction.

\begin{tabular}{|c|c|c|c|c|c|c|c|}
\hline \multirow{2}{*}{ Source } & \multirow{2}{*}{$\mathrm{df}$} & \multicolumn{2}{|c|}{ Assay 1} & \multicolumn{2}{|c|}{ Assay 2} & \multicolumn{2}{|c|}{ Assay 3} \\
\hline & & MS & $P$ & MS & $P$ & MS & $P$ \\
\hline Parasite identity & 2 & 0.158 & 0.010 & 0.131 & 0.064 & 0.084 & 0.075 \\
\hline Replicate line (identity) & 6 & 0.015 & ns & 0.029 & ns & 0.020 & ns \\
\hline $\mathrm{DR} / \mathrm{CR}$ & 1 & 0.004 & $\mathrm{~ns}$ & 0.064 & $\mathrm{~ns}$ & 0.003 & ns \\
\hline $\mathrm{DR} / \mathrm{CR} \times$ identity & 2 & 0.034 & ns & 0.133 & 0.007 & 0.124 & 0.089 \\
\hline Error & 6 & 0.015 & & 0.010 & & 0.033 & \\
\hline
\end{tabular}

ns: $P>0.1$.

Table 2. Correlation coefficients describing the relationship between mean direct and correlated response to selection for parasites evolving on three host clones (K3, K4, and K8) in the three assays. Correlations are based on the mean responses calculated for each parasite replicate line $(N=3)$. Variation in these relationships was tested in an ANCOVA, with the correlated response as dependent variable, and parasite identity ( $2 \mathrm{df})$ and the direct response (DR; $1 \mathrm{df}$ ) and their interaction ( $2 \mathrm{df}$ ) as explanatory variables. These ANCOVA models were significant in assay 2 (see text), but not in assay $1\left(F_{5,8}=0.54, n s\right)$ and $3\left(F_{5,8}=0.60, n s\right)$.

\begin{tabular}{llrc}
\hline & \multicolumn{3}{l}{ Parasite identity } \\
\cline { 2 - 4 } & $\mathrm{K} 4$ & \multicolumn{1}{c}{ K3 } & K8 \\
\hline Assay 1 & 0.97 & 0.10 & -0.45 \\
Assay 2 & 1.00 & -0.96 & -0.96 \\
Assay 3 & 0.02 & -0.35 & -0.49 \\
\hline
\end{tabular}

\section{PATTERNS AT DAY 25 POST INOCULATION (ASSAY 2)}

The ANOVA revealed a significant interaction between parasite identity and the type of response to selection (Table 1). In particular, the mean direct response was positive for parasites from clones $\mathrm{K} 3\left(t_{8}=2.23, P=0.0562\right)$ and $\mathrm{K} 8\left(t_{8}=2.43, P=0.0414\right)$, whereas that for K4 parasites was clearly not significantly different from $0\left(\mathrm{t}_{8}=-0.29, P=0.7802\right.$; Fig. 3$)$.

\section{Costs of Adaptation}

All parasites showed positive correlated responses on $\mathrm{K} 3$ (mean: $0.35 \pm 0.16)$ and on $\mathrm{K} 8(0.28 \pm 0.10)$ hosts. $\mathrm{K} 3$ and $\mathrm{K} 8$ parasites regressed on $\mathrm{K} 4$ hosts $(-0.12 \pm 0.14$; Figs. $4 \mathrm{~A}-\mathrm{C})$, indicating a cost of adaptation. K4 parasites did not seem to advance on their own host or regress on $\mathrm{K} 3$ or K8 hosts.

The relationship between direct and average correlated responses to selection varied with parasite identity. For parasite replicate lines evolving on $\mathrm{K} 3$ and $\mathrm{K} 8$, a stronger increase in infection on their own host was associated with a smaller average correlated increase on foreign hosts. However, for K4 parasites, this relationship was positive: stronger (i.e., less negative) direct responses were associated with stronger correlated responses to selection (ANCOVA: parasite identity $\times$ direct response interaction: $F_{2,3}=173.59, P=0.0008$; Fig. 5). Thus, again, adaptation appeared to be costly on K3 or K8, but not on K4 hosts. The corresponding analyses for assays 1 and 3 showed qualitatively similar, although nonsignificant, patterns. Thus, in all three assays, there was a positive relationship between direct and correlated response

Table 3. Analysis of deviance of prevalence as a function of host clone, host subclone, and resident versus nonresident parasite (RES/NONRES) in the three assays. Host clone was tested against subclone, RES/NONRES against the RES/NONRES $\times$ host clone interaction, the other terms against the error. Quasi- $F$ tests were based on mean deviances (MD; $2 \times \log$-likelihood ratio/degrees of freedom), analogous to mean squares in an ANOVA.

\begin{tabular}{|c|c|c|c|c|c|c|c|}
\hline \multirow{2}{*}{ Source } & \multirow{2}{*}{ df } & \multicolumn{2}{|c|}{ Assay 1} & \multicolumn{2}{|c|}{ Assay 2} & \multicolumn{2}{|c|}{ Assay 3} \\
\hline & & MD & $P$ & MD & $P$ & MD & $P$ \\
\hline Host clone & 2 & 84.7 & 0.074 & 134.7 & $<0.001$ & 125.0 & 0.032 \\
\hline Host subclone (clone) & 6 & 20.4 & 0.035 & 10.3 & 0.047 & 19.3 & 0.041 \\
\hline RES/NONRES & 1 & 4.6 & ns & 4.2 & $0.025^{*}$ & 0.04 & ns \\
\hline RES/NONRES $\times$ clone & 2 & 7.1 & $\mathrm{~ns}$ & 0.11 & ns & 14.2 & 0.100 \\
\hline Error & 6 & 4.1 & & 2.33 & & 4.1 & \\
\hline
\end{tabular}

ns: $P>0.1 ;{ }^{*}$ nonsignificant $\left(F_{1,8}=2.35, P=0.1638\right)$ in a simplified model with RES/NONRES $\times$ clone interaction pooled with the error term. 


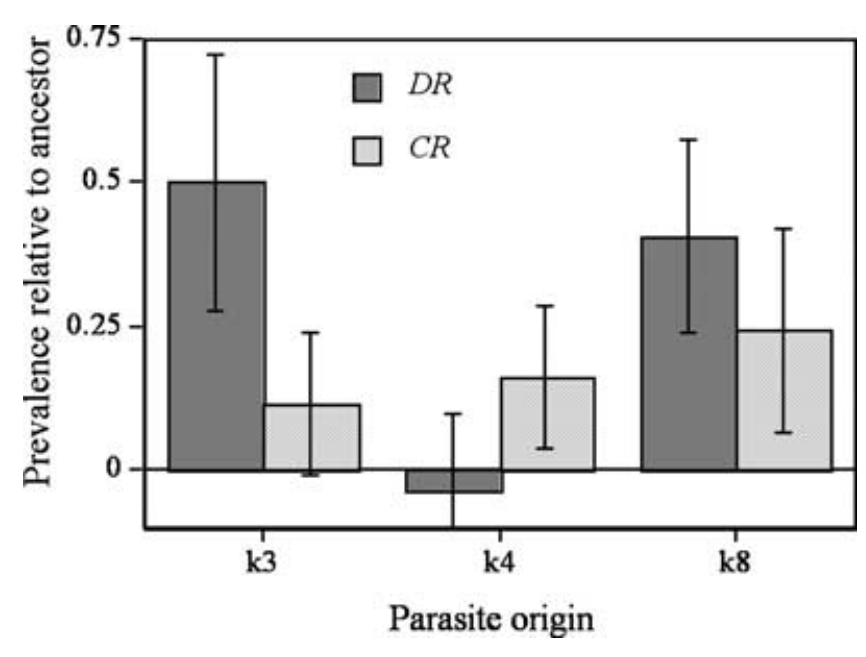

Figure 3. Mean ( $\pm 1 \mathrm{SE}$ ) direct (DR) and correlated (CR) responses to selection in assay 2 , shown for the three parasite identities. Positive values indicate a higher infection success of evolved parasites relative to the ancestral parasites. DR: Averages taken over combinations of parasite replicate line and host subclone (DR: $N=9$; CR: $N=18$ ).

for $\mathrm{K} 4$ parasites, whereas the correlations for $\mathrm{K} 3$ and $\mathrm{K} 8$ parasites were negative in almost all cases (Table 2). An ANOVA of these correlation coefficients revealed a significant effect of parasite identity $\left(F_{2,6}=6.34, P=0.0331\right)$.

\section{Genotype-Specificity: Resident versus Nonresident Parasites}

Statistical evidence for the evolution of host genotype specificity depended on the type of analysis. For all the three pairwise comparisons, the parasite identity $\times$ host genotype interaction terms in the Analyses of Deviance were nonsignificant $\left(F_{1,24}<1.48\right.$, $P>0.2$ ). Note, however, the different tendencies for the different combinations of host and parasite. In the K3-K8 combination (Fig. 4B), both direct and correlated responses to selection of $\mathrm{K} 3$ and $\mathrm{K} 8$ parasites were positive and nearly identical, resulting in essentially parallel reaction norms, with a slightly overall higher infection success of $\mathrm{K} 8$ parasites on both hosts (Fig. 4E). For the other two combinations (K8-K4, K3-K4), the direct responses to selection exceeded the correlated responses (Figs. 4A,C), which translated into crossing reaction norms and a marginal advantage of resident over nonresident parasites (Figs. 4D,F).

Overall, resident parasites produced more infections than did nonresidents on all three host clones. However, the ANODEV based on prevalences of residents and nonresidents on each subclone revealed a significant resident advantage only in the full, but not in the simplified model (Table 3). In an alternative approach, analogous to pairwise competition trials, we calculated the mean difference in prevalence between all possible pairs of resident and nonresident parasite lines $(N=54)$. We then further averaged to
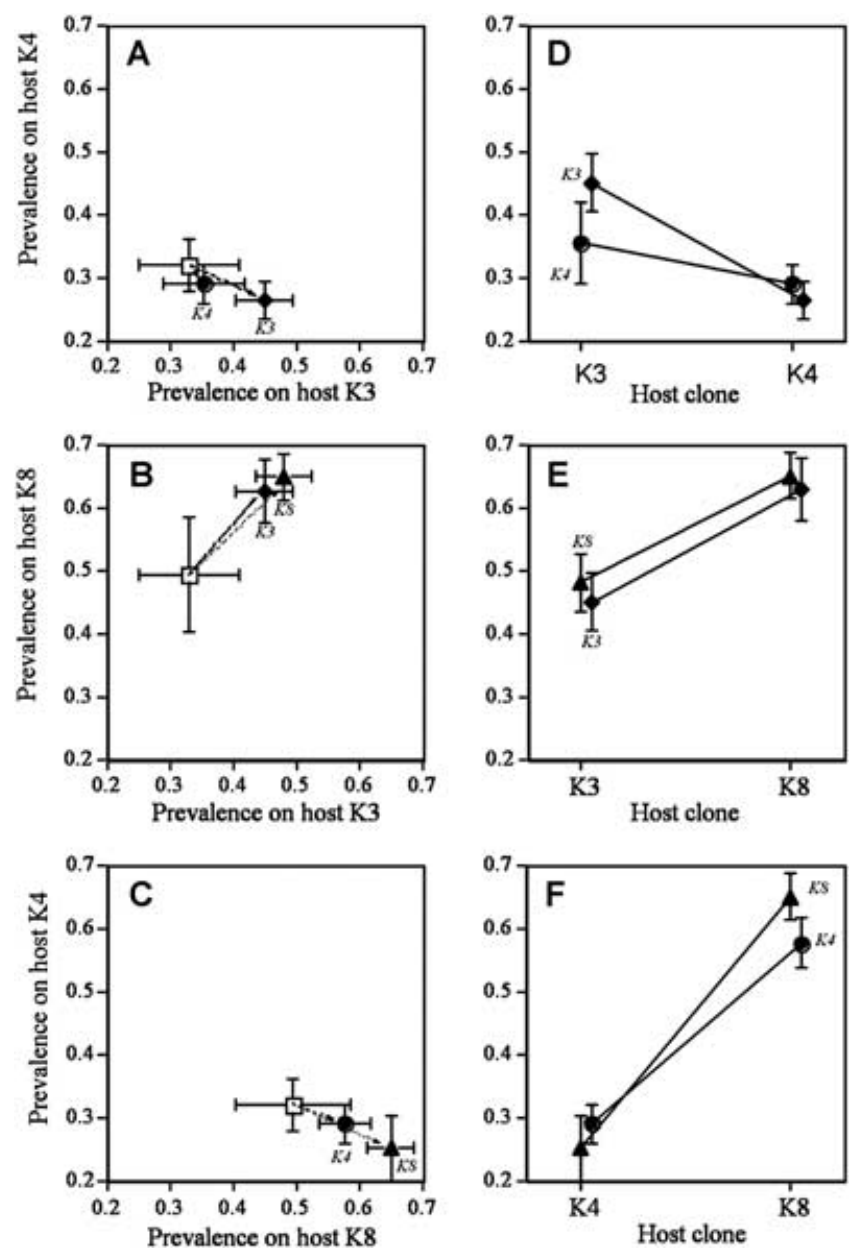

Figure 4. Mean ( $\pm 1 \mathrm{SE}$ ) infection success in pairwise comparisons of host clones, analogous to Figure 1. (A-C) Performance of evolved and ancestral parasites, with arrows indicating the advance or regress relative to the ancestral parasites. (D-E) Reaction norms of evolved parasites on the two host clones.

obtain the means per parasite replicate line. These means were significantly larger than $0\left(t_{8}=2.36, P=0.0461\right)$, indicating an overall resident advantage. Thus, when considering (combinations of) parasite lines as units of replication a consistent general trend of specialization emerged in this assay (Fig. 6), supporting the results from the ANODEV (Table 2).

\section{Discussion}

Assay 2 revealed an overall positive response to selection of this parasite, both on the genotypes on which it evolved (direct response) and the genotypes on which it did not evolve (correlated response). This demonstrates that adaptation of parasites to novel hosts does not necessarily lead to a decrease in performance on other (novel) hosts (Gould 1979; Agrawal 2000; Buckling and Rainey 2002). For example, trade-offs may be overruled by adaptation to features common to all novel hosts, at least during early 


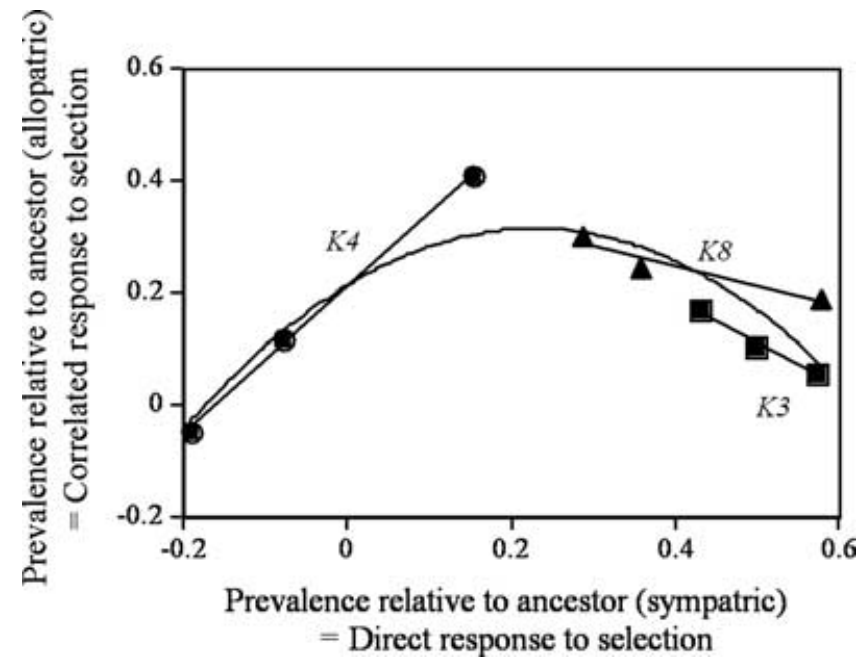

Figure 5. Relationship between mean direct (DR) and correlated (CR) response to selection for the three parasite identities. Each point represents a replicate selection line. A negative relationship between DR and CR is consistent with the idea of pleiotropic costs of adaptation. Note that for this to occur the CR does not have to be negative. Straight lines represent linear regressions through each group of three points. The dashed line represents a second-order polynomial regression through all points, illustrating a potential hump-shaped general relationship between DR and CR.

stages of selection (Joshi and Thompson 1995). Remember that the three host clones tested in our experiment share the same parental strains and thus a common genetic background to which the parasites may have adapted. However, adaptation may also occur to a novel laboratory environment or to the experimental protocol. The weekly replacement of a fraction of our long-term cultures with fresh medium produced low, but constant levels of host di-

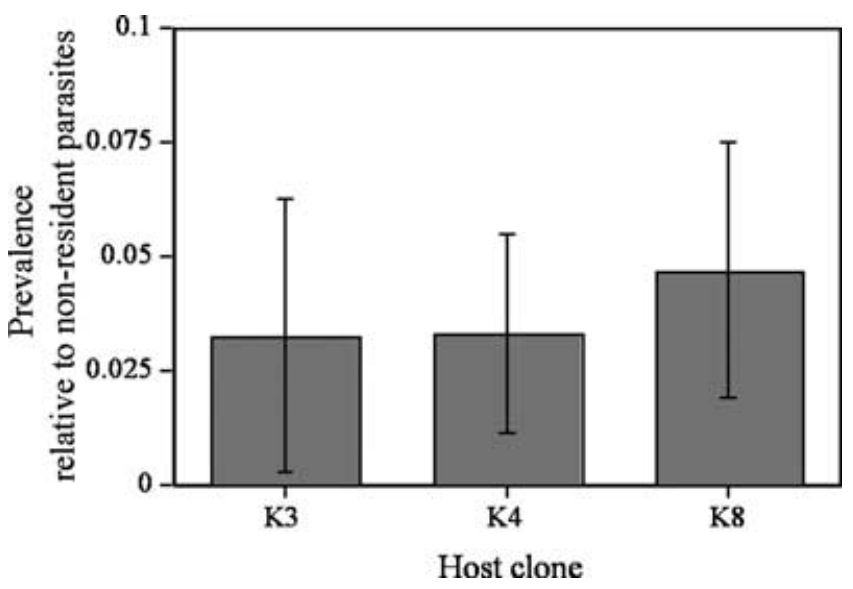

Figure 6. Mean ( $\pm 1 \mathrm{SE}$ ) difference in infection success between resident and nonresident parasites on the three host clones. Averages taken over mean differences between parasite replicate lines and nonresident identities $(N=3$ resident replicate lines $\times$ 2 nonresident identities $=6$ ). Positive values indicate a resident advantage. vision, and thereby opportunities for vertical transmission. This may have imposed a general selection pressure for enhanced efficacy of vertical transmission and thus explain the overall higher prevalences of evolved versus ancestral parasites in assay 2 .

Nonetheless, positive correlated responses to selection do not preclude host genotype-specific adaptation. Patterns of specialization can arise as long as the direct response to selection of the resident parasite exceeds the correlated response of the nonresident parasites (Whitlock 1996; Kawecki 1998; Buckling and Rainey 2002; Kassen 2002; MacLean and Bell 2002). That is, it does not even require a positive direct response to selection. This point is illustrated by the K4 parasites: they did not progress on their own host clone, but their direct response was nonetheless less negative than the correlated responses of K3 and K8 parasites. Conversely, the positive correlated response of K4 parasites was smaller than the direct responses of $\mathrm{K} 3$ and $\mathrm{K} 8$, respectively. As a result, there were consistent trends of a resident advantage on each host clone.

An instructive way to test for costs of adaptation is to examine the relationship between direct and correlated responses to selection (Turner and Elena 2000; MacLean and Bell 2002). A negative association indicates a cost, presumably due to antagonistic pleiotropy: Genes conferring a stronger advance in the selection environment are expected to cause a stronger regress in foreign environments. Even though their correlated response was, on average, positive, K3 and K8 parasites showed such a negative correlation. Replicate lines of these parasites that advanced more on their own hosts advanced relatively less on foreign hosts (Fig. 5), which is thus consistent with the idea of antagonistic pleiotropy and a cost of adaptation (see also Turner and Elena 2000). For K4 parasites, however, this relationship was positive, suggesting the absence of such a cost. There are two ways to interpret these patterns. First, they suggest that evolutionary trajectories are convergent on some hosts, but not on others. Parasites evolving on $\mathrm{K} 3$ and $\mathrm{K} 8$ host genotypes produced nearly identical responses to selection (even though the two hosts differed in resistance and were thus not genetically identical) that were qualitatively different from those of parasites evolving on host clone K4. Moreover, if adaptation to the $\mathrm{K} 4$ clone is indeed cost-free, the trajectory for K4 parasites in Figure 5 implies that further adaptation to this clone will generate universally superior generalist parasites. Alternatively, costs may arise as soon as K4 parasites begin to truly advance on their own host and produce positive direct responses to selection. We do not have a ready explanation for the low level of adaptation to the K4 host. One possibility could be its high level of resistance (relative to the two other host clones), which could lead to smaller parasite populations and thus a reduced evolutionary potential.

These results raise the question whether different host genetic backgrounds generate different relationships between direct and correlated responses to selection, or whether there exists a single 
relationship (here: hump-shaped, shown as dashed line in Fig. 5), common to all backgrounds. Obviously, the answer to this question is important for the evolution of genotype $\times$ genotype interactions and the evolution of genotype-specific parasites. To investigate in more detail the shape of these curves (Fig. 5), we are currently running an adaptation experiment with a larger number of parasite replicate lines per host genetic background.

\section{LIMITATIONS AND PERSPECTIVES}

This was our first attempt to measure evolutionary change in this system, and several lessons can be learned. First, some of our interpretations (e.g., concerning the patterns of local adaptation) had relatively weak statistical support, in part due to unexpected variation in resistance among supposedly genetically identical subclones. Whether this reflects uncontrolled environmental or true genetic variation, remains to be investigated. Further, only three parasite replicate lines per host genetic background were tested, which limits generalizations about host genotype-specific differentiation. In principle, these problems can be solved by increasing replication at the relevant level.

Second, it was not a priori clear which parasite fitness component had been under selection. We therefore measured prevalence at different time points post inoculation. As a result, we obtained different patterns in the different assays (Fig. 2). As outlined above, an optimistic interpretation is that assay 2 (day $25 \mathrm{PI}$ ) reflects most closely the conditions in the long-term selection lines and therefore integrates best over the different fitness components (vertical and horizontal transmission, virulence). Indeed, this assay provided the best resolution between evolved and ancestral parasites and the clearest indication of host-specific adaptation. However, although assays should measure fitness under the conditions of selection, they should not allow evolutionary changes to occur during the assay. This point is important when dealing with organisms with a short generation time and/or genetically variable assay populations. In this respect, we feel uncomfortable about the time until the final assay (62 days PI), which may have provided ample opportunity for selection to operate. Thus, an optimal compromise is required between a short-term assay measuring only a single fitness component (e.g., infectivity) and a longer-term assay allowing epidemiological dynamics to integrate over several components. For example, the risk of evolutionary change in our assays could have been minimized by isolating single strains from each parasite replicate line and using these strains rather than (potentially) genetically diverse cocktails in the assays.

A third point concerns the choice of hosts from uninfected control lines for the adaptation assay. Preliminary tests for one of the three host clones had not revealed significant differences in resistance between hosts from evolved and control lines (Nidelet 2004). We had therefore decided to consider only adaptation by the parasite and treated host genotype as a constant environment.
However, if coevolution occurs in our microcosms, the patterns of adaptation found on naive hosts may represent the adaptation past, but not necessarily the coevolutionary status quo. In fact, a follow-up experiment one year later (Lohse et al. 2006) indicated a pattern of maladaptation of parasites when testing them on evolved hosts. More generally, this suggests that genotype $\times$ genotype interactions do not evolve towards a stable equilibrium (as perhaps expected for genotype $\times$ environment interactions in abiotic environments), but rather change dynamically over coevolutionary time scales. The sign and magnitude of the interactions and patterns of adaptation then depend on the relative evolutionary rates of the two players (Gandon 2002). With microbial systems, it is often possible to track these dynamics by comparing host and parasites from different time points of their coevolutionary history (Morgan et al. 2005).

Finally, our microcosms represent a set of spatially isolated populations, each harboring a single host genotype. More realistically, populations are connected by some degree of gene flow so that regular exposure to different hosts may select for generalist parasites. Similar to our results, Legros and Koella (2005) demonstrated the evolution of specialization of a microsporidian parasite to isofemale lines of the mosquito Aedes aegypti. However, generalist parasites selected on a mix of these lines had a higher mean fitness than the specialists. Thus, even if costs of adaptation exist, specialization may not be the only possible evolutionary outcome (see also Turner and Elena 2000).

\section{Conclusions}

We think that our study, despite its limitations, illustrates the usefulness of the approach of direct/correlated responses to selection for studying adaptation in host-parasite interactions. It can help us to understand why genotype $\times$ genotype interactions do or do not evolve, or why they are asymmetric. The causes of these interactions (antagonistic pleiotropy and/or mutation accumulation) can be assessed in a simple way by testing for a relationship between direct and correlated responses to selection. Our results indicate that these relationships can be very different for different host genetic backgrounds (Fig. 5), suggesting that adaptation to some hosts may even be cost free. More generally, this kind of test is based on the comparison of replicate selection lines and therefore also provides information about the repeatability and congruence of (co)evolutionary trajectories.

\section{ACKNOWLEDGMENTS}

We thank S. Fellous, J. Koella, J. Shykoff and two anonymous referees for helpful comments and discussions and L. Millot for maintaining the selection lines. This project was financed by a research grant "ACI Jeunes Chercheuses et Jeunes Chercheurs" (Ministère de Recherche, France) to OK. 


\section{LITERATURE CITED}

Agrawal, A. A. 2000. Host-range evolution: adaptation and trade-offs in fitness of mites on alternative hosts. Ecology 81:500-508.

Agrawal, A. F., and C. M. Lively. 2002. Infection genetics: gene-for-gene versus matching-alleles models and all points in between. Evol. Ecol. Res. 4:79-90.

Amann, R., N. Springer, W. Ludwig, H.-D. Görtz, and K.-H. Schlaifer. 1991. Identification in situ and phylogeny of uncultured bacterial endosymbionts. Nature 351:161-164.

Bell, G. 1997. Selection: the mechanism of evolution. Chapman \& Hall, New York.

Bergelson, J., G. Dwyer, and J. Emerson. 2001. Models and data on plantenemy coevolution. Ann. Rev. Genet. 35:469-499.

Buckling, A., and P. B. Rainey. 2002. Antagonistic coevolution between a bacterium and a bacteriophage. Proc. R. Soc. Lond. B 269:931936.

De Wit, P. J. G. M. 1997. Pathogen avirulence and plant resistance: a key role for recognition. Trends Plant Sci. 2:452-458.

Dybdahl, M., and A. Storfer. 2003. Parasite local adaptation: red Queen versus Suicide King. Trends Ecol. Evol. 18:523-530.

Ebert, D. 1998. Experimental evolution of parasites. Science 282:1432-1435.

Fels, D., and O. Kaltz. 2006. Temperature-dependent transmission and latency of Holospora undulata, a micronucleus-specific parasite of the ciliate Paramecium caudatum. Proc. R. Soc. Lond. B 273:1031-1038.

Fokin, S. I. 2004. Bacterial endocytobionts of ciliophora and their interactions with the host cell. Int. Rev. Cytol 236:181-249.

Frank, S. A. 1994. Recognition and polymorphism in host-parasite genetics. Phil. Trans. R. Soc. Lond. Ser. B 346:283-293.

Fry, J. D. 1990. Trade-offs in fitness on different hosts: evidence from a selection experiment with a phytophagous mite. Am. Nat. 136:569-580.

Futuyma, D. J., and G. Moreno. 1988. The role of ecological speciation. Annu. Rev. Ecol. Syst. 19:207-233.

Gandon, S. 2002. Local adaptation and the geometry of host-parasite coevolution. Ecol. Lett. 5:246-256.

Görtz, H.-D. 1988. Paramecium. Springer, Berlin.

Görtz, H.-D., and J. Dieckmann. 1980. Life cycle and infectivity of Holospora elegans Haffkine, a micronucleus-specific symbiont of Paramecium caudatum (Ehrenberg). Protistologia 16:591-603.

Gould, F. 1979. Rapid host range evolution in a population of the phytophagous mite Tetranychus urticae Koch. Evolution 33:791-802.

Joshi, A., and J. N. Thompson. 1995. Trade-offs and the evolution of host specialization. Evol. Ecol. 9:82-92.

Kaltz, O., and J. C. Koella. 2003. Host growth conditions regulate the plasticity of horizontal and vertical transmission in Holospora undulata, a bacterial parasite of the protozoan Paramecium caudatum. Evolution 57:14891497.

Kaltz, O., and J. A. Shykoff. 1998. Local adaptation in host-parasite systems. Heredity 81:361-370.

Karban, R. 1989. Fine-scale adaptation of herbivorous thrips to individual host plants. Nature 340:60-61.

Kassen, R. 2002. The experimental evolution of specialists, generalists and the maintenance of diversity. J. Evol. Biol. 15:173-190.

Kawecki, T. J. 1998. Red Queen meets Santa Rosalia: arms races and the evolution of host specialization in organisms with plastic life styles. Am. Nat. 152:635-651.

Kawecki, T. J., and D. Ebert. 2004. Conceptual issues in local adaptation. Ecol. Lett. 7:1225-1241.

Lajeunesse, M. J., and M. R. Forbes. 2002. Host range and local parasite adaptation. Proc. R. Soc. Lond. B 269:703-710.

Lambrechts, L., S. Fellous, and J. C. Koella. 2006. Coevolutionary interactions between host and parasite genotypes. Trends Parasitol 22: $12-16$.

Legros, M., and J. C. Koella. 2005. Experimental specialization of microsporidian parasites to their mosquito host. ESEB X Conference, Krakow, Poland.

Levins, R. 1968. Evolution in changing environments. Princeton Univ. Press, Princeton, NJ.

Lohse, K., A. Gutierrez, and O. Kaltz. 2006. Experimental evolution of resistance in Paramecium caudatum against the bacterial parasite Holospora undulata. Evolution 60:1177-1186.

MacLean, R. C., and G. Bell. 2002. Experimental adaptive radiation in Pseudomonas. Am. Nat. 160:569-581.

Millot, L., and O. Kaltz. 2006. Cryopreservation of Holospora undulata, a bacterial parasite of the ciliate Paramecium caudatum. Cryobiology 62:161165.

Morand, S., S. D. Manning, and M. E. Woolhouse. 1996. Parasite-host coevolution and geographic patterns of parasite infectivity and host susceptibility. Proc. R. Soc. Lond. B 263:119-128.

Morgan, A. D., S. Gandon, and A. Buckling. 2005. The effect of migration on local adaptation in a coevolving host-parasite system. Nature 437:253256.

Nidelet, T. 2004. Evolution des interactions génotype-génotpe dans un système hôte-parasite, Paramecium caudatum et Holospora undulata. Dept. Diversité du Vivant. Université Pierre \& Marie Curie, Paris, France.

Restif, O., and O. Kaltz. 2006. Condition-dependent virulence in a horizontally and vertically transmitted bacterial parasite. Oikos 114:148158.

SAS. 1996. SAS/STAT User's guide, Release 6.11. SAS Institute, Cary, NC.

- 2003. JMP statistics and graphics guide (version 5.0.1.2). SAS Institute, Cary, NC.

Schmid-Hempel, P., and D. Ebert. 2003. On the evolutionary ecology of specific immune defence. Trends Ecol. Evol. 18:27-32.

Thompson, J. N., and J. J. Burdon. 1992. Gene-for-gene coevolution between plants and parasites. Nature 360:121-125.

Turner, P. E., and S. F. Elena. 2000. Cost of host radiation in an RNA virus. Genetics 156:1465-1470.

Whitlock, M. C. 1996. The Red Queen beats the Jack-of-all-Trades: the limitations on the evolution of phenotypic plasticity and niche breadth. Am. Nat. 148:S65-S77.

Wichtermann, R. T. 1986. The biology of Paramecium. Plenum Press, New York.

Wiemann, M., and H.-D. Görtz. 1989. Release of the endonucleobiotic bacterium Holospora elegans from its host cell Paramecium caudatum. Eur. J. Protistol. 25:100-108.

Associate Editor: J. Shykoff 